УДК 004.932.4:519.233.5

doi: $10.32620 /$ aktt.2018.6.13

\author{
А. С. ГРИГОРЬЕВА, В. В. АБРАМОВА, В. В. ЛУКИН, \\ Н. В. КОЖЕМЯКИНА, К. Д. АБРАМОВ
}

Национальный аэрокосмический университет им. Н. Е. Жуковского «ХАИ», Украина

\title{
СПЕЦИФИКА ОЦЕНИВАНИЯ ХАРАКТЕРИСТИК СЛОЖНЫХ ПОМЕХ С УЧЕТОМ МЕЖКАНАЛЬНОЙ КОРРЕЛЯЦИИ НА ГИПЕРСПЕКТРАЛЬНЫХ ИЗОБРАЖЕНИЯХ
}

\begin{abstract}
Предметом изучения в статье является скаттерограммный метод автоматического оченивания характеристик сложных помех на многоканальных изображениях. Целью является рассмотрение проблемных вопросов, связанных с адаптацией данного метода для работы с гиперспектральными изображениями. Задачи: исследовать влияние способа формирования групп канальных изображений, подлежащих совместной обработке, на точность исследуемого метода оценивания и стабильность его работы; сформулировать рекомендации по выбору изображений, подлежащчих совместной обработке и способу объединения их в группы. При решении поставленных задач были использованы методы робастного оченивания параметров сигналов, а также методы спектрально-корреляционного и регрессионного анализа. Получены следуюшче результаты. Рассмотрены три возможных способа формирования групп из трех канальных изображений: 1) совлестная обработка изображений, принадлежащзих соседним каналам; 2) совместная обработка изображений, имеющчих наивыстие коэффициенть взаимной корреляции; 3) совместная обработка изображений, имеющчих наиболее низкие коэффициенты взаимной корреляции. Показано, что в случае, если коэффициенты взаимной корреляции изображений, входящих в группу, низкие, а сами изображения характеризуются сложной структурой, возможно существенное снижение точности метода, вплоть до полной утрать его работоспособности. При формировании групп из соседних изображений и из изображений с наивысшими коэффиџиентами межсканальной корреляции метод демонстрирует достаточно высокие точность и стабильность, причем значения оценок параметров помех для этих вариантов отличаются слабо. Выводы. Способ формирования группь существенно влияет не только на точность, но и на работоспособность рассматриваемого метода оченивания, и для обеспечения более высокой надежности его работы целесообразно объединять в группу изображения с достаточно высокими коэффициентами межканальной корреляичи. Однако поскольку точность метода в ситуаичях, когда в группу объединяются изображения, принадлежащие соседним каналам, и изображения с наивысшими коэффициентами корреляции, отличаются незначительно, с иелью сохранения высокого быстродействия метода, группь изображений для их совместной обработки рекомендуется формировать из изображений, полученных в соседних спектральных зонах.
\end{abstract}

Ключевые слова: дистанциинное зондирование; гиперспектральные изображения; межканальная коррелячия; сложные помехи; автоматическое оченивание характеристик помех

\section{Введение}

Дистанционное зондирование можно определить как метод оценивания свойств объектов на земной поверхности, в котором используются данные, полученные при помощи воздушных летательных аппаратов и искусственных спутников Земли [1]. Данные дистанционного зондирования Земли (Д33) являются эффективным инструментом, который позволяет оперативно и детально исследовать состояние окружающей среды и получать объективную картину мира, что обусловило их широкое использование в сельском и лесном хозяйстве, в задачах экологического мониторинга, гидрологии, картографирования, метеорологии и др. $[1,2]$.
Системы Д3 различают по используемому спектральному диапазону, по типу приёмника регистрируемого излучения, а также по методу зондирования (активный или пассивный) $[1,3]$. В зависимости от используемого диапазона электромагнитного излучения выделяют системы оптического диапазона (объединяющего видимую, ближнюю и среднюю инфракрасные (ИК) области спектра) и радиочастотные (радиолокационные). Кроме того, различают системы космического и авиационного базирования. [1 - 3]. Однако независимо от типа системы Д3, общей особенностью изображений, формируемых этими системами, является присутствие на них помех и искажений, которые ухудшают как визуальное качество полученных снимков, так и резуль-

() А. С. Григорьева, В. В. Абрамова, В. В. Лукин, Н. В. Кожемякина, К. Д. Абрамов 
таты их обработки. Характеристики искажающих помех следует учитывать на разных этапах обработки: при сжатии данных Д3 $[4,5]$, при извлечении полезной информации (классификация, поиск границ, объектов и т.п.) [6, 7]. В некоторых случаях целесообразно предварительное подавление помех при помощи специальных фильтров [8], однако для правильного выбора фильтра и настройки его параметров также необходимо знание статистических характеристик помех. Для реальных систем предсказать характеристики помех априори часто невозможно, поскольку они являются функцией большого количества случайных факторов $[1,3]$. В связи с этим информацию о характеристиках помех получают непосредственно по обрабатываемому изображению при помощи специальных автоматических методов $[1,9,10]$.

Следует отметить, что искажающие помехи, как правило, имеют сложную структуру, характеризующуюся наличием сигнально-независимой и сигнально-зависимой компонент с явным преобладанием последней. Кроме того, эти помехи могут быть в значительной степени пространственно коррелированными [10 - 12]. Несмотря на достаточно большое количество методов автоматического оценивания характеристик помех, разработанных к настоящему моменту [10 - 16], среди них нет универсального решения, способного обеспечить приемлемую точность оценивания [17] и быстродействие во всех практических ситуациях, поэтому задача разработки новых методов и усовершенствования уже существующих решений не утрачивает своей актуальности.

\section{Постановка задачи}

В последние несколько десятилетий особое распространение получили многоканальные системы формирования изображений. В таких системах изображение одного и того же участка земной поверхности формируется различными способами (на разных частотах, поляризациях, с разными углами визирования и т.п.), что позволяет обеспечить более надежную классификацию и интерпретацию данных. Количество каналов в таких системах колеблется от двух-трех до нескольких сотен [1 - 3]. Среди многоканальных систем особое место занимают так называемые гиперспектральные системы [18 - 21], характеризующиеся весьма малой шириной спектральных полос (зон), используемых для получения компонентных изображений. Именно обработке изображений, полученных гиперспектральными системами, являющимися наиболее сложными, будет уделено внимание в данной работе.
Как было отмечено ранее, изображения дистанционного зондирования искажены сложными помехами, содержащими сигнально-независимую и сигнально-зависимую компоненты. Упрощенная модель такого изображения может быть записана в следующем виде:

$$
\mathrm{g}(\mathrm{m}, \mathrm{l})=\mathrm{n}_{\mathrm{sd}}(\mathrm{m}, 1 ; \mathrm{s}(\mathrm{m}, \mathrm{l}))+\mathrm{n}_{\mathrm{a}}(\mathrm{m}, \mathrm{l}),
$$

где $\mathrm{s}(\mathrm{m}, \mathrm{l})$ - истинное (незашумленное) изображение;

$\mathrm{n}_{\mathrm{a}}(\mathrm{m}, \mathrm{l})$ - компонент, описывающий влияние сигнально-независимого (аддитивного) шума, который обычно является нормально распределенным и характеризуется дисперсией $\sigma_{\mathrm{a}}^{2}$;

$\mathrm{n}_{\mathrm{sd}}(\mathrm{m}, \mathrm{l})$ - функционал, описывающий влияние сигнально-зависимого шума.

Для гиперспектральных изображений сигнально-зависимая компонента, как правило, имеет квазипуассоновский характер, поэтому для них модель (1) может быть переписана в виде:

$$
\mathrm{g}(\mathrm{m}, \mathrm{l})=\mathrm{n}_{\mathrm{p}}(\mathrm{m}, \mathrm{l} ; \mathrm{s}(\mathrm{m}, \mathrm{l}))+\mathrm{n}_{\mathrm{a}}(\mathrm{m}, \mathrm{l}),
$$

где $\mathrm{n}_{\mathrm{p}}(\mathrm{m}, \mathrm{l})$ - функционал описывающий влияние квази-пуассоновского шума с коэффициентом усиления $\mathrm{k}$.

Оценивание характеристик помех на гиперспектральных изображениях является достаточно сложной задачей, поскольку эти изображения, как правило, имеют сложную структуру и содержат множество областей с текстурами, малоразмерными объектами и градиентными изменениями интенсивности и малое число однородных участков. Поэтому для работы с такими изображениями должны использоваться методы оценивания, обладающие высокой робастностью по отношению к влиянию информационной составляющей изображения.

Еще одной особенностью гиперспектральных изображений является высокая степень межканальной корреляции. Коэффициенты межканальной корреляции для соседних изображений могут быть очень близкими к единице, что позволяет использовать совместную обработку нескольких изображений для уменьшения влияния информационной компоненты изображения на результат оценивания характеристик помех на нем.

Для оценивания характеристик помех такого вида был недавно разработан и успешно протестирован на гиперспектральных изображениях метод [22]. Этот метод включает в себя следующие основные этапы.

1. Выбор трех каналов многоканального изображения (в случае цветного трехканального изображения используются все его каналы).

2. Кластеризация канальных изображений и получение оценок среднего в кластерах $\left(\mathrm{I}_{1 \mathrm{loc}_{\mathrm{cl}_{1}}}, \mathrm{I}_{2 \mathrm{loc}_{\mathrm{cl}_{2}}}, \mathrm{I}_{3 \mathrm{loc}_{\mathrm{cl}_{3}}}\right.$, где $\mathrm{cl}_{1}, \mathrm{cl}_{2}, \mathrm{cl}_{3}$ - номера кла- 
стеров каждого из компонентных (канальных) изображений; $\mathrm{cl}_{1}=1 \ldots \mathrm{n}_{1}, \mathrm{cl}_{2}=1 \ldots \mathrm{n}_{2}, \mathrm{cl}_{3}=1 \ldots \mathrm{n}_{3}$, де $\mathrm{n}_{1}, \mathrm{n}_{2}$, $\mathrm{n}_{3}$ - количество кластеров для каждого из компонентных изображений соответственно) согласно методике, описанной в [23].

3. Получение разностных изображений для всех возможных пар каналов без повторений.

4. Получение оценок дисперсии в кластерах для каждого из разностных изображений посредством применения метода [24]. Поскольку для разных канальных изображений границы и центры кластеров отличаются, поиск оценок дисперсии осуществляется отдельно по каждой из имеющихся карт кластеризации. В результате выполнения этого этапа для каждого кластера образуется девять оценок дисперсии (по три оценки, полученных по трем разностным изображениям, рассчитанным для трех различных карт кластеризации).

5. Для получения оценок дисперсии в кластеpax, которые отвечают не разностным, а исходным канальным изображениям, необходимо составить систему линейных уравнений, содержащую три оценки дисперсии, полученные по одной кластеризационной карте, как показано в [22]:

$$
\left\{\begin{array}{l}
\hat{\sigma}_{\mathrm{a} 1 \mathrm{i}}^{2}+\hat{\sigma}_{\mathrm{a} 2 \mathrm{i}}^{2}=\hat{\sigma}_{\mathrm{a} 12 \mathrm{i}}^{2} \\
\hat{\sigma}_{\mathrm{a} 1 \mathrm{i}}^{2}+\hat{\sigma}_{\mathrm{a} 3 \mathrm{i}}^{2}=\hat{\sigma}_{\mathrm{a} 13 \mathrm{i}}^{2}, \\
\hat{\sigma}_{\mathrm{a} 2 \mathrm{i}}^{2}+\hat{\sigma}_{\mathrm{a} 3 \mathrm{i}}^{2}=\hat{\sigma}_{\mathrm{a} 23 \mathrm{i}}^{2}
\end{array}\right.
$$

где i - номер кластеризационной карты, который, в свою очередь, соответствует номеру канала, по которому она была получена. Решение этой системы уравнений будет иметь следующий вид:

$$
\left\{\begin{array}{l}
\hat{\sigma}_{\mathrm{a} 1 \mathrm{i}}^{2}=0,5\left(\hat{\sigma}_{\mathrm{a} 12 \mathrm{i}}^{2}+\hat{\sigma}_{\mathrm{a} 13 \mathrm{i}}^{2}-\hat{\sigma}_{\mathrm{a} 23 \mathrm{i}}^{2}\right) \\
\hat{\sigma}_{\mathrm{a} 2 \mathrm{i}}^{2}=0,5\left(\hat{\sigma}_{\mathrm{a} 12 \mathrm{i}}^{2}-\hat{\sigma}_{\mathrm{a} 13 \mathrm{i}}^{2}+\hat{\sigma}_{\mathrm{a} 23 \mathrm{i}}^{2}\right) \\
\hat{\sigma}_{\mathrm{a} 3 \mathrm{i}}^{2}=0,5\left(\hat{\sigma}_{\mathrm{a} 23 \mathrm{i}}^{2}-\hat{\sigma}_{\mathrm{a} 12 \mathrm{i}}^{2}+\hat{\sigma}_{\mathrm{a} 13 \mathrm{i}}^{2}\right)
\end{array} .\right.
$$

6. Для получения финальных оценок параметров помех, присутствующих на исходных канальных изображениях, необходимо провести вписывание регрессионной прямой по опорным точкам скаттерограммы локальных оценок дисперсии и среднего. При этом для получения параметров помех для первого канала используются опорные точки с координатами ( $\left.\mathrm{I}_{1 \operatorname{loc}_{\mathrm{cl}_{1}}}, \hat{\sigma}_{\mathrm{a} 11}^{2}\right)$, для второго - $\left(\mathrm{I}_{1 \mathrm{loc}_{\mathrm{cl}_{2}}}\right.$, $\left.\hat{\sigma}_{\mathrm{a} 22}^{2}\right)$, для третьего - $\left(\mathrm{I}_{1 \mathrm{loc}_{\mathrm{cl}_{3}}}, \hat{\sigma}_{\mathrm{a} 33}^{2}\right)$, то есть для каждого канала используются оценки центров кластеров и наборы оценок дисперсии, полученные с использованием соответствующей этому каналу кластеризационной карты. Вписывание выполняется с использованием дважды взвешенного метода наименьших квадратов (ДВМНК) [23].
7. Параметры вписанных прямых принимаются в качестве оценок параметров сигнальнонезависимой и сигнально-зависимой компонент помex.

Вышеописанный метод оценивания характеристик помех с учетом межканальной корреляции предполагает совместную обработку трех канальных изображений. В случае цветного изображения используются все его каналы, однако если изображение имеет большее количество каналов, возможны различные варианты выбора трех каналов для совместной обработки. При этом логично предположить, что от того, какие именно компонентные изображения обрабатываются совместно, будет зависеть результат оценивания характеристик помех на многоканальном изображении.

В частности, гиперспектральные изображения, полученные системой AVIRIS, содержат 224 канала каждое. Следовательно, для каждого канального изображения существует $\mathrm{C}_{223}^{2}$ возможных варианта выбора двух дополнительных изображений для совместной обработки (С - число сочетаний), что составляет 24753 варианта. Разумеется, перебор всех возможных вариантов требует значительного времени и вычислительных ресурсов и является неприемлемым для практики решением. Поэтому выбор изображений для совместной обработки должен осуществляться по определенным критериям.

Одним из таких критериев может быть коэффициент межканальной корреляции между совместно обрабатываемыми изображениями. Однако вопрос о том, насколько сильно этот параметр влияет на точность оценивания характеристик помех на изображениях, до сих пор не выяснен и требует дополнительного рассмотрения. Поэтому целью данной работы является исследование влияния степени межканальной корреляции между совместно обрабатываемыми изображениями на результат оценивания характеристик помех на многоканальном изображении. Этот анализ будет проводиться для гиперспектральных изображений, полученных системой AVIRIS.

\section{Описание эксперимента}

В ходе исследований было использовано два гиперспектральных изображения AVIRIS: Cuprite, приведенное на рис. 1, и Lunar Lake, несколько каналов которого показаны на рис. 2.

Как видим, эти изображения имеют достаточно сложную структуру и содержат множество границ, мелких деталей и текстурных областей. При этом хорошо заметно, что информационные составляющие различных каналов имеют достаточно высокую 


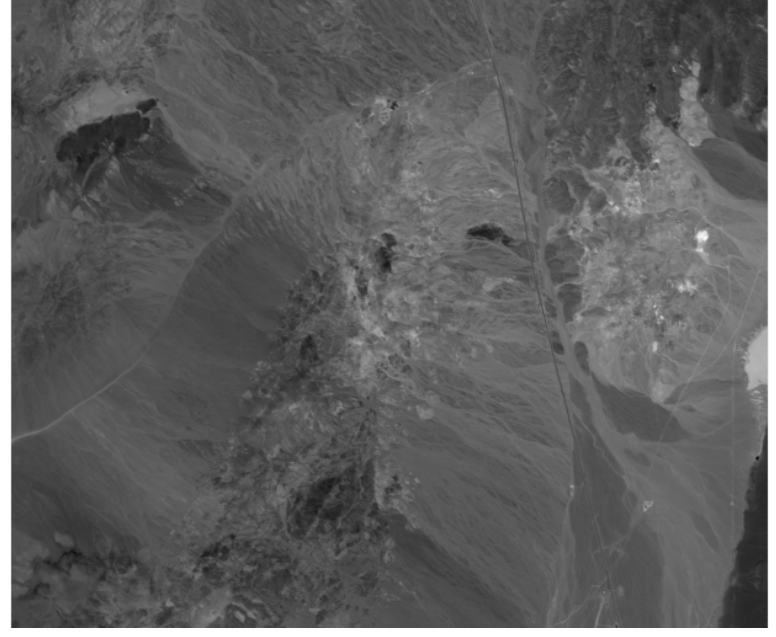

Рис. 1. Изображение Cuprite (122 канал), полученное системой AVIRIS

степень взаимной корреляции, в то время как уровень помех для различных каналов существенно отличается, причем для отдельных каналов этот

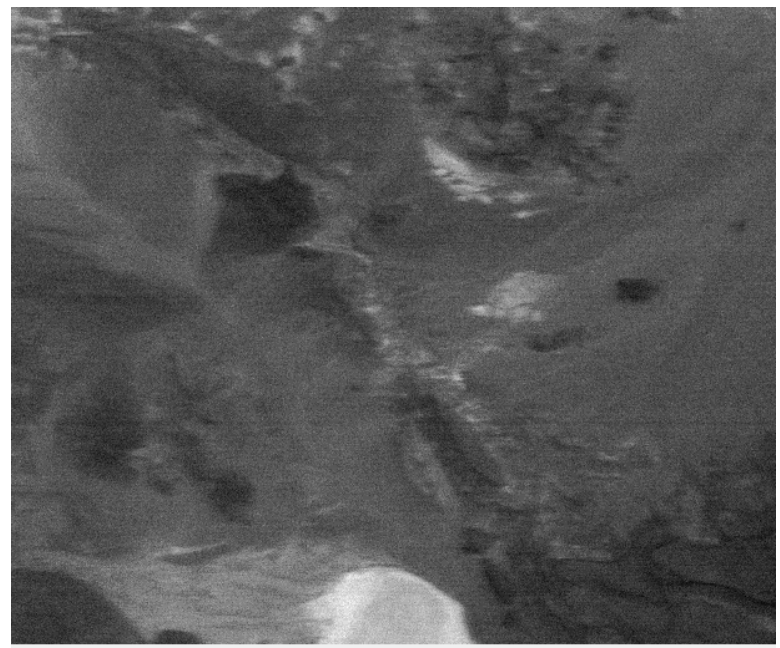

a

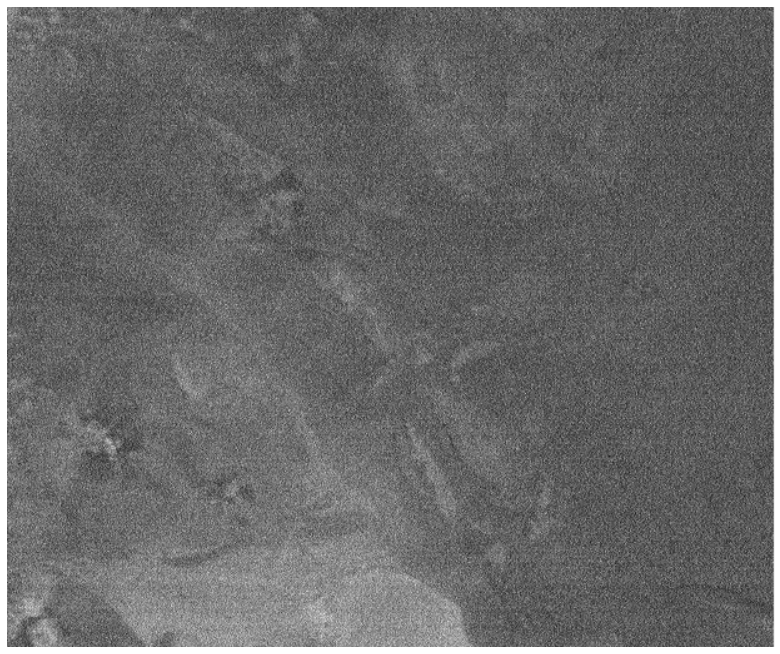

B уровень является достаточно высоким.

Согласно результатам исследований, проведенных учеными из разных научных групп $[3,25]$, в большинстве случав наивысшие значения коэффициентов межканальной корреляции наблюдаются для соседних каналов многоканального изображения. Поэтому на первом этапе исследований формирование групп изображений для совместной обработки производилось по принципу соседства, т. е. для получения оценки характеристик помех для nтого канала, где $\mathrm{n}=2 \ldots 223$, в состав группы совместно обрабатываемых каналов входили каналы с номерами n-1, n, n+1; для получения оценок характеристик помех для первого канала использовалась группа 224, 1, 2, а для канала № 224 - группа 223, $224,1$.

На втором этапе было принято решение исследовать степень влияния корреляции между компонентными изображениями, входящими в состав группы, на точность оценивания характеристик помех. В ходе исследований были рассмотрены два

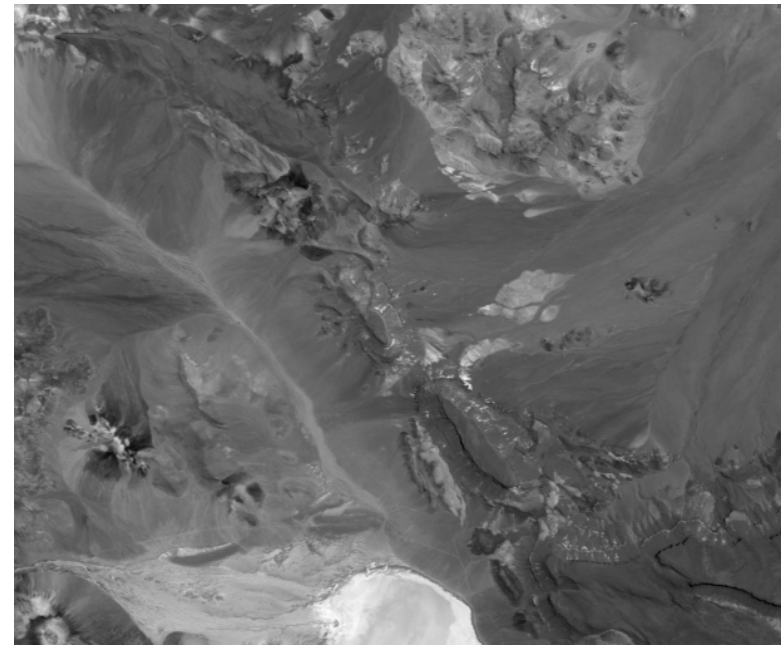

6

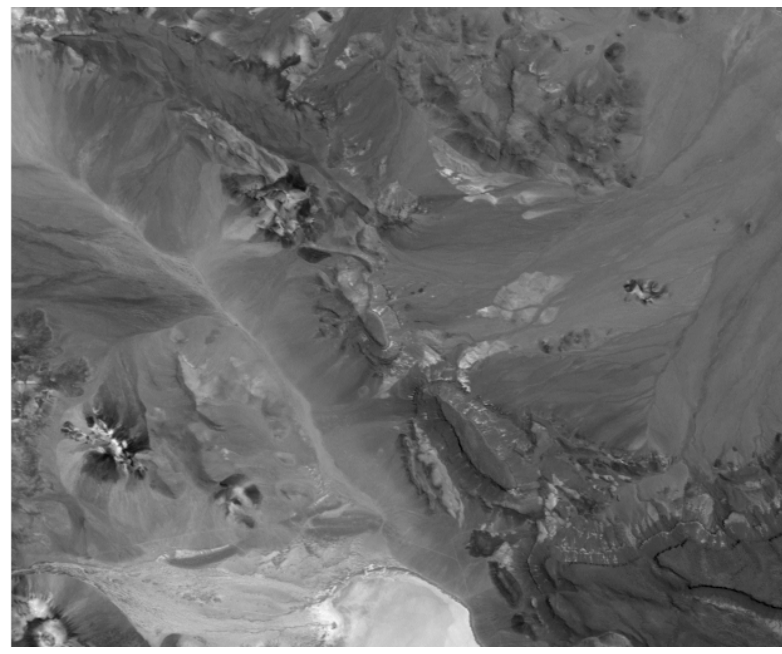

Рис. 2. Различные каналы гиперспектрального изображения Lunar Lake: 2-й (a), 121-й (б), 163-й (в), 204-й (г) 
способа формирования групп: «наилучший», при котором в состав группы включались изображения с максимальными коэффициентами взаимной корреляции и «наихудший», при котором в группу включались изображения с наиболее низкими значениями коэффициентов взаимной корреляции.

При анализе полученных результатов в качестве эталонных были использованы оценки параметров шума, полученные методом [25]. Данный метод основан на оценивании максимального правдоподобия характеристик шума и информационной составляющей изображения и является одним из наиболее точных среди существующих методов оценивания характеристик сложных помех. Недостатком метода [25] является его существенная вычислительная сложность, обуславливающая низкое быстродействие данного метода.

\section{Анализ результатов}

На рис. 3 показаны коэффициенты взаимной корреляции для всех каналов гиперспектральных изображений Cuprite и Lunar Lake; номера каналов отложены вдоль горизонтальных осей. Как видно из представленных данных, в большинстве случаев коэффициенты межканальной корреляции являются достаточно высокими, причем наибольшие значения этих коэффициентов наблюдаются, в основном, для соседних каналов. Однако имеется ряд каналов, для которых характерны весьма низкие коэффициенты взаимной корреляции с другими каналами: это группа каналов в районе 120-го и в районе 160-го, для которых характерен наиболее высокий уровень помех и искажений. Интересным является тот факт, что коэффициенты межканальной корреляции для изображений Cuprite и Lunar Lake существенно отличаются, однако полосы «плохих» каналов имеются для обоих изображений и примерно совпадают.

На рис. 4 представлены гистограммы, показывающие, сколько раз каждый из каналов был использован при формировании групп каналов для совместной обработки в «наилучшем» и «наихудшем» вариантах. Как видим, в «наилучшем» варианте большая часть канальных изображений вошла в

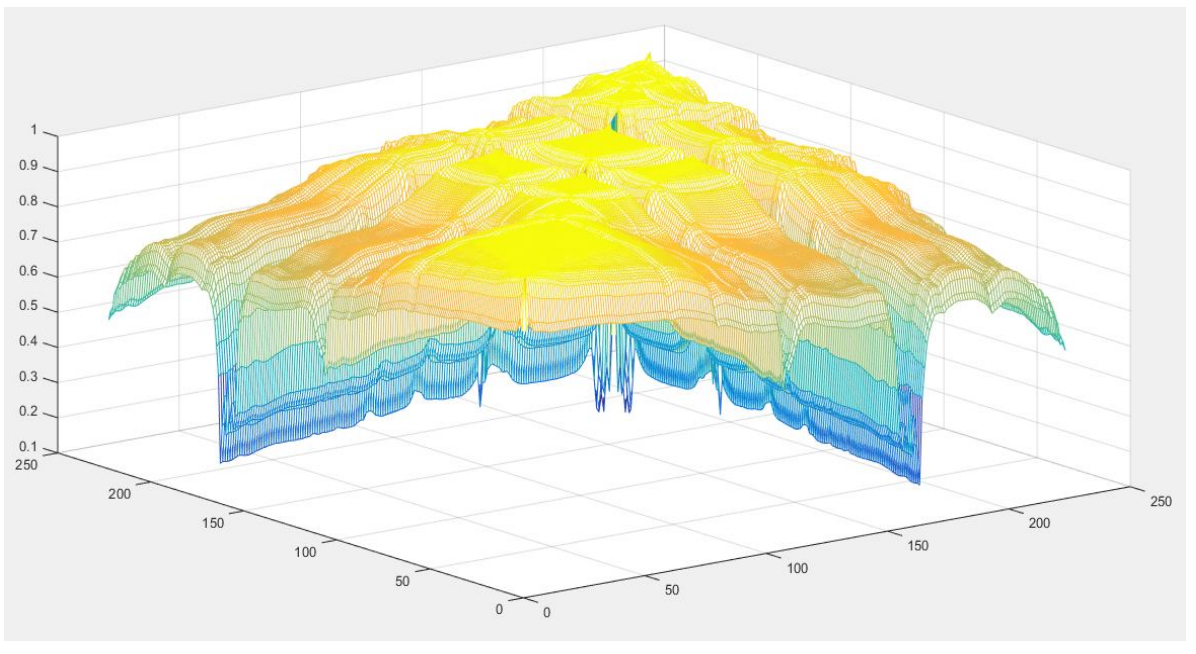

a

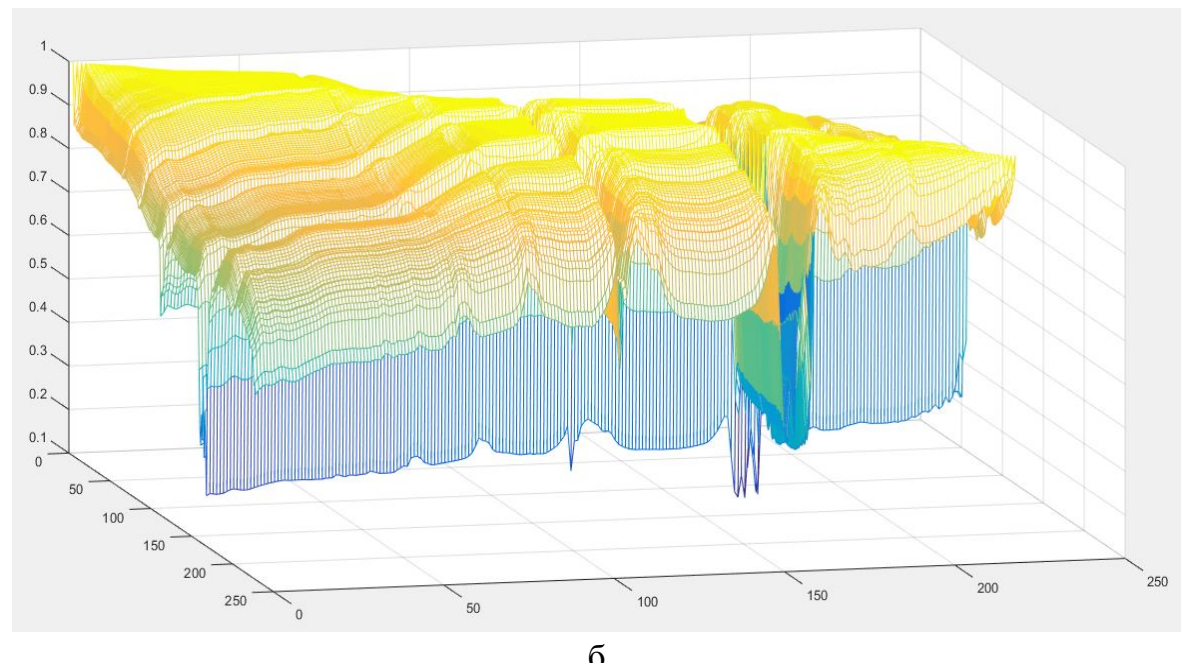

6

Рис. 3. Коэффициенты межканальной корреляции для изображений Cuprite (a) и Lunar Lake (б) 

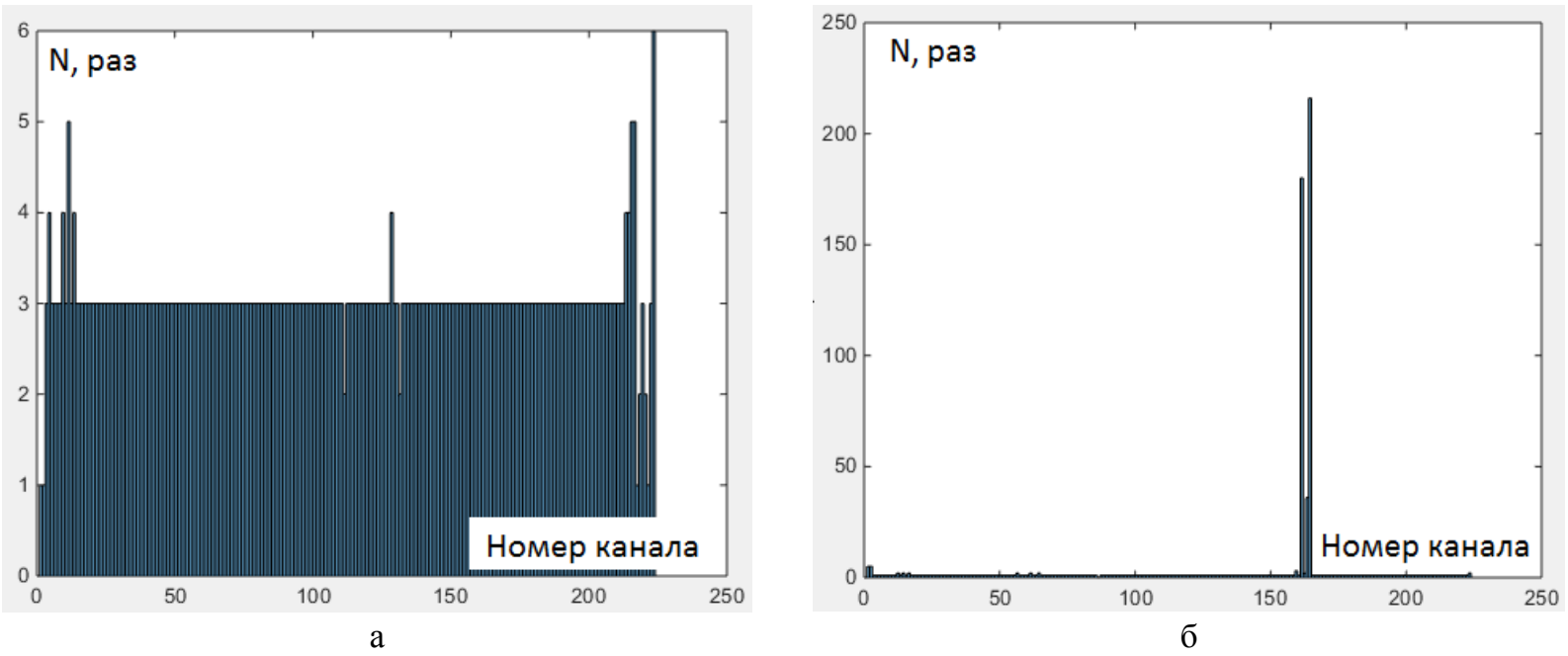

Рис. 4. Гистограммы использования каналов изображения Cuprite при формировании их групп для совместной обработки в «наилучшем» (а) и «наихудшем» (б) вариантах

состав трех групп, при этом в подавляющем большинстве ситуаций это были собственно группа, созданная для оценивания параметров помех в данном канале, а также группы, при помощи которых производилось оценивание характеристик помех в каналах по соседству. Наиболее используемым ока- зался канал № 224, который вошел в состав 6 групп.

Рассмотрим теперь гистограмму для «наихудшего» случая, приведенную на рис. 4, б. Очевидно, что разброс коэффициентов использования каналов в этом случае значительно выше, чем в «наилучшем»: имеются два очевидных «лидера», это каналы
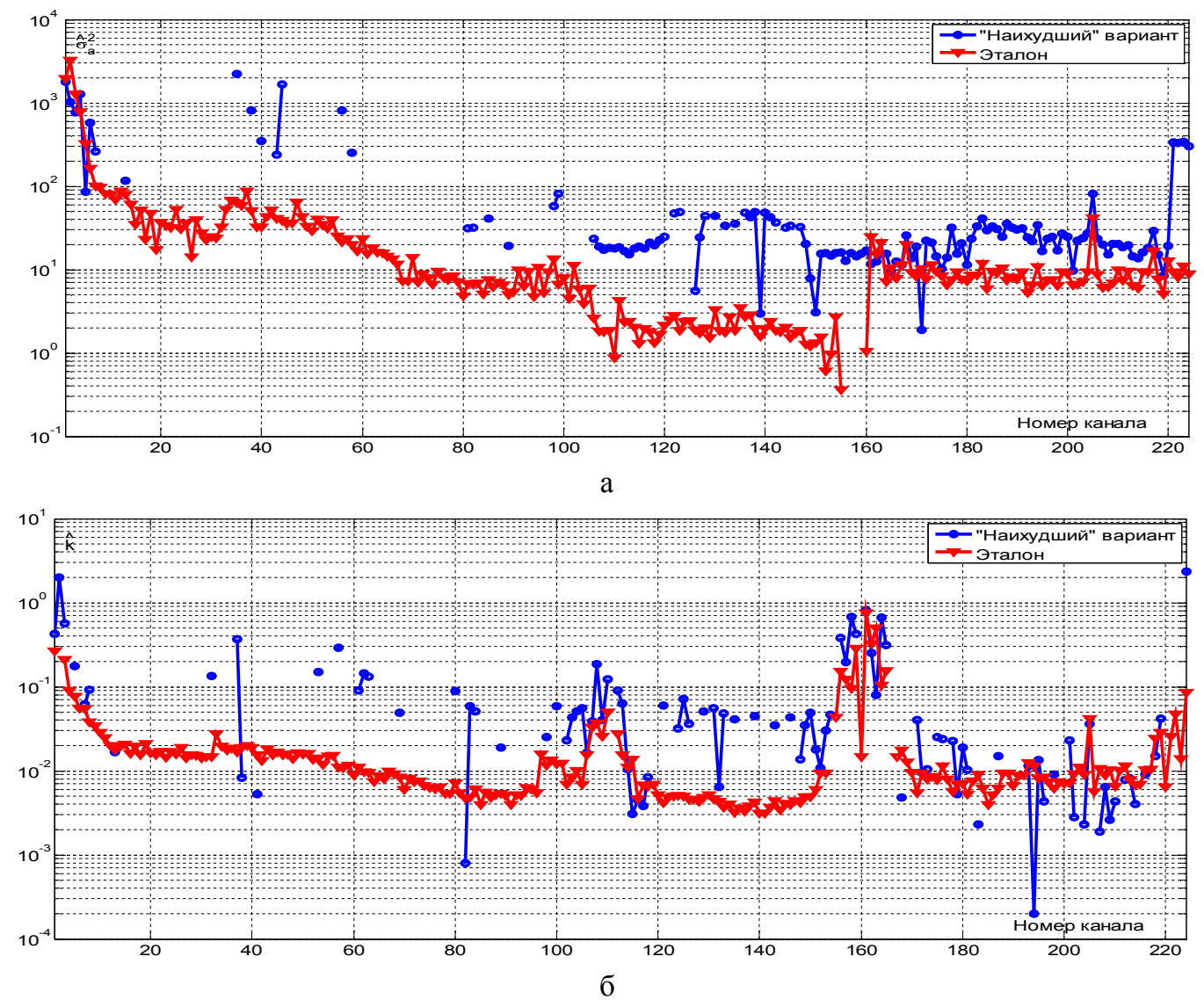

Рис. 5. Оценки дисперсии аддитивного шума (а) и коэффициента усиления квази-пуассоновского шума (б), полученные для изображения Cuprite при использовании «наихудшего» варианта формирования групп изображений для совместной обработки 
№ 161 и 164, которые вошли в состав почти всех групп. Как уже было указано ранее, изображения, полученные в этих каналах, характеризуются наиболее низким качеством, что и обусловило низкие коэффициенты их взаимной корреляции с изображениями, полученными в остальных каналах гиперспектрального изображения.

На рис. 5 показаны оценки параметров шума на изображении Cuprite, полученные при формировании групп в соответствии с «наихудшим» вариантом; для сравнения приведены также результаты для эталонного метода. Как видим, кривые для обоих методов содержат разрывы. В случае эталонного метода наличие разрывов связано с представлением данных в логарифмическом масштабе, при котором игнорируются нули и отрицательные значения. Что касается исследуемого метода, то для него разрывы на графиках соответствуют тем изображениям, для которых метод не смог детектировать однородные участки в количестве, достаточном для получения оценки параметров помех. Как видно из представленных результатов, исследуемый метод позволил получить оценки только для половины каналов, однако эти оценки существенно (на 1-2 порядка) отличаются от оценок, полученных при помощи эталон- ного метода.

Рассмотрим результаты оценивания характеристик помех (рис. 6), полученные при «наилучшем» варианте формировании групп, а также при формировании групп из изображений, полученных в соседних каналах. Как видим, в отличие от «наихудшего» варианта, в данном случае оценки параметров шума были получены для всех изображений и кривые на рис. 6 не содержат разрывов (исключением являются несколько канальных изображений, для которых оценка параметра сигнально-зависимого шума оказалась равной нулю).

Если сравнивать между собою результаты для вариантов «по соседству» и «наилучший», то несложно заметить, что эти кривые практически совпадают и обе расположены достаточно близко к кривой для эталонного метода. Исключение составляют несколько компонентных изображений, для которых заметны существенные отличия между оценками параметров шума, полученными при помощи исследуемого и эталонного методов. Следует отметить, что более низкая точность исследуемого метода по сравнению с эталонным может быть частично скомпенсирована его существенно (в несколько раз) более высоким быстродействием.

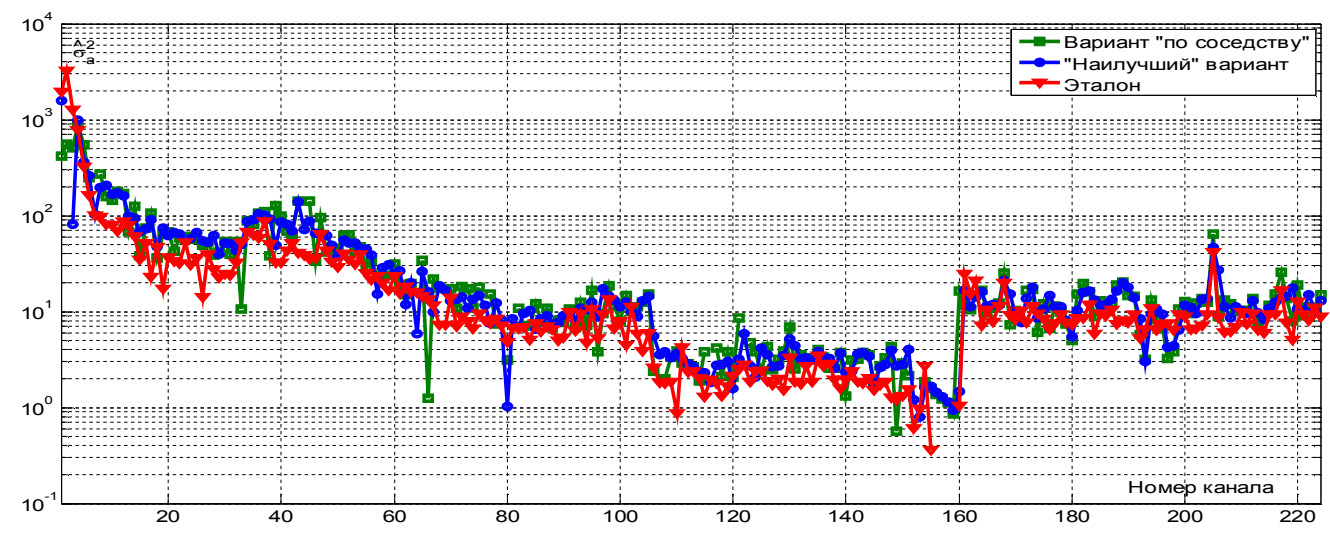

a

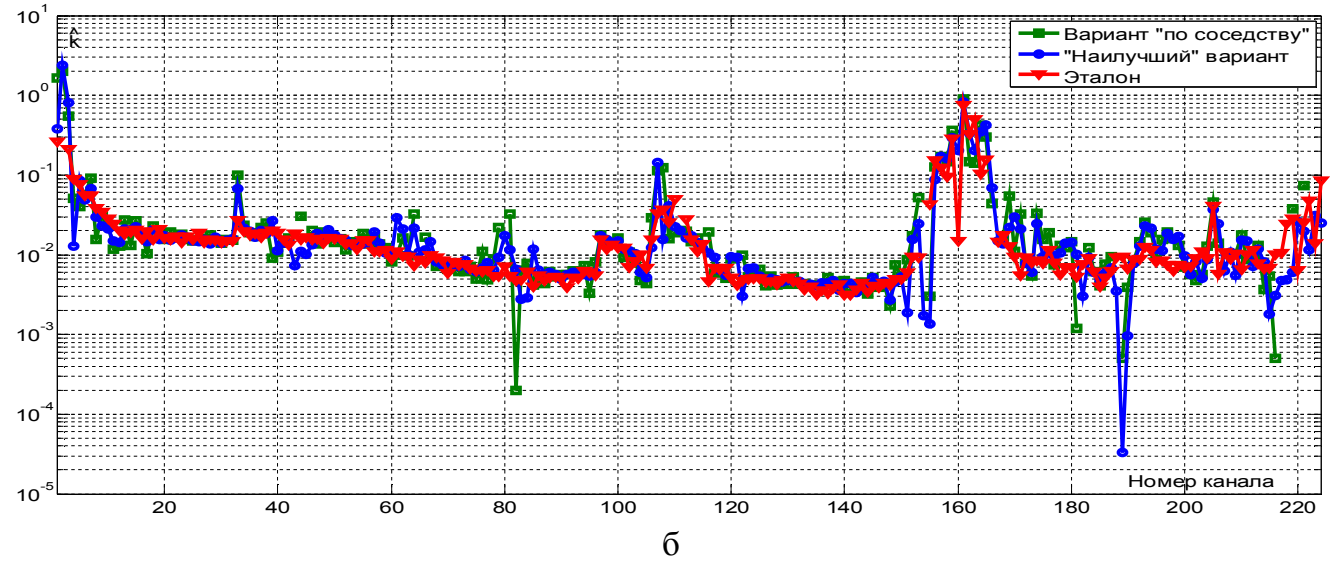

Рис. 6. Оценки дисперсии аддитивного шума (а) и коэффициента усиления квази-пуассоновского шума (б), полученные для изображения Cuprite при формировании групп по соседству (a) и при использовании каналов с наивысшими коэффициентами взаимной корреляции 
С одной стороны, полученные результаты свидетельствуют о том, что состав группы совместно обрабатываемых изображений может оказывать существенное влияние на точность исследуемого метода оценивания характеристик помех. В случае, если коэффициенты взаимной корреляции изображений, входящих в группу, низкие, а сами изображения характеризуются сложной структурой (содержат множество текстур и мелких деталей и малый процент однородных областей), возможно существенное снижение точности метода, вплоть до полной утраты его работоспособности, что свидетельствует о необходимости предварительного расчета и последующего учета коэффициентов взаимной корреляции изображений при формировании групп. С другой стороны, процесс расчета коэффициентов межканальной корреляции при наличии большого числа каналов является достаточно продолжительной операцией, что способствует снижению быстродействия метода, а это крайне нежелательно, поскольку быстродействие метода является одним из основных его преимуществ.

Компромиссным вариантом для практического использования может являться вариант формирования групп изображений по принципу соседства без дополнительного учета степени взаимной корреляции между изображениями, входящими в состав группы. Как видно из представленных данных (рис. 6), степень взаимной корреляции изображений, полученных в соседних каналах, в большинстве случаев является достаточно высокой, и точность оценивания параметров помех на изображениях при использовании групп, сформированных из соседних изображений, незначительно отличается от результатов, полученных при формировании групп из изображений с наивысшими коэффициентами взаимной корреляции.

\section{Заключение}

В статье рассмотрены особенности применения метода оценивания характеристик сложных помех с учетом межканальной корреляции [22] к гиперспектральным изображениям, полученным системой AVIRIS. Эти особенности заключаются в существовании большого количества вариантов формирования групп из трех компонентных изображений, подлежащих совместной обработке с целью получения оценок характеристик помех. Были рассмотрены три возможных способа формирования таких трехканальных групп:

1) совместная обработка изображений, принадлежащих соседним каналам;

2) совместная обработка изображений, имеющих наивысшие коэффициенты взаимной корреляции;
3) совместная обработка изображений, имеющих наиболее низкие коэффициенты взаимной корреляции.

В ходе проведения исследований было показано, что способ формирования группы существенно влияет не только на точность, но и на работоспособность рассматриваемого метода оценивания, и для обеспечения более высокой надежности работы метода целесообразно объединять в группу изображения с достаточно высокими коэффициентами межканальной корреляции. Показано, что при объединении в группу изображений, полученных в соседних каналах, точность оценивания параметров шума незначительно отличается от точности в случае, когда в состав группы включаются изображения с максимальными коэффициентами взаимной корреляции. Следовательно, с целью сохранения высокого быстродействия метода, группы изображений для их совместной обработки рекомендуется формировать из изображений, полученных в соседних спектральных зонах.

\section{Литература}

1. Шовенгердт, Р. А. Дистаниионное зондирование. Модели и методы обработки изображений [Текст]/ Р. А. Шовенгердт. - М. : Техносфера. $-2010 .-560 \mathrm{c}$.

2. Интегращия гетерогенной пространственной информачии для решения задач поиска нефти и газа [Электронный ресурс] / М. Попов, С. Станкевич, С. Марков и др. // Российский научный электронный журнал «Электронные библиотеки». - 2013. - T. 16, № 2. - Режим доступа: http://www.elbib.ru. - 14.11.2018.

3. Kerekes, J.P. Optical Sensor Technology[Text] / J. P. Kerekes // The SAGE Handbook of Remote Sensing. - London, UK : SAGE Publications, 2009. - P. $95-107$.

4. Christophe, E. Hyperspectral Data Compression Tradeoff in Optical Remote Sensing / E. Christophe // Advances in Signal Processing and Exploitation Techniques. 8th ed. - Springer; Berlin Heidelberg. - 2011. - P. 9-29.

5. Bekhtin, Y. S. Adaptive wavelet codec for noisy image compression / Y. S. Bekhtin // Proceedings of the 9th East-West Design and Test Symposium, September 2011, Sevastopol, Ukraine. - Sevastopol, 2011. - P. $184-188$.

6. Image Classification Techniques in Remote Sensing [Electronic Resource]. - Available at: https://gisgeography.com/image-classificationtechniques-remote-sensing/. - 1.10.2018.

7. Edge-guided image object detection in multiscale segmentation for high-resolution remotely sensed imagery [Text] / Y. Hu, J. Chen, D. Pan, Z. Hao // IEEE Transactions on Geoscience and Remote Sensing. -2016. - Vol. 54, No. 8. - P. 4702-4711. 
8. Zhong, P. Multiple-spectral-band CRFs for denoising junk bands of hyperspectral imagery [Text] / P. Zhong, R. Wang // IEEE Transactions on Geoscience and Remote Sensing. - 2013. - No. 51(4). - P. $2269-$ 2275 .

9. Modeling and estimation of signal-dependent noise in hyperspectral imagery [Text] / J. Meola, M. T. Eismann, R. L. Moses, J. N. Ash // Applied Optics. - 2011. - No. 50(21). - P. 3829-3846.

10. A non-parametric approach for the estimation of intensity-frequency dependent noise [Text] / M. Colom, M. Lebrun, A. Buades, J. M. Morel // IEEE International Conference on Image Processing (ICIP), 27-30 October 2014, Paris, France. - Paris, 2014. - P. 4261-4265.

11. Goosens, B. Removal of Correlated Noise by Modeling the Signal of Interest in the Wavelet Domain [Text] / B. Goossens, A. Pizurica, P. Wilfried // IEEE Transactions on Image Processing. - 2009. - Vol. 18, Issue 6. - P. $1-14$.

12. Cocianu, C. Neural Architectures for Correlated Noise Removal in Image Processing [Electronic Resource] / C. Cocianu, A. Stan // Mathematical Problems in Engineering. - 2016. - Vol. 2016. - Article ID: 6153749. - 14 p. - Available at: https://www.hindawi.com/journals/mpe/ 2016/6153749/. - 14.11.2018.

13. Automatic estimation and removal of noise from a single image [Text] / C. Liu, R. Szeliski, S.B. Kang, C.L. Zitnick, W.T. Freeman // IEEE Transactions on Pattern Analysis and Machine Intelligence. - 2008. - No. 30(2). - P. 299-314.

14. A comparative study on linear regression based noise estimation for hyperspectral imagery [Text] / L. Gao, Q. Du, B. Zhang, W. Yang, Y. Wu // IEEE Journal of Selected Topics in Applied Earth Observations and Remote Sensing. - 2013. - No. 6(2). - P. 488-498.

15. Jin, X. Noise parameter estimation for poisson corrupted images using variance stabilization transforms [Text] / X. Jin, Z. Xu, K. Hirakawa // IEEE Trans Image Process. - 2014. - No. 23(3). - P. 1329 - 1339.

16. Savant, R. V. Estimation of noise parameters for captured image [Electronic Resource] / R. V. Savant, D. Pradhan // IEEE International Conference on Engineering and Technology (ICETECH), March 17 - 18, Coimbature, India. - Coimbature, 2016. Available at: https://ieeexplore.ieee.org/document/ 7569405. - 20.11.2018

17. On required accuracy of mixed noise parameter estimation for image enhancement via denoising [Electronic Resource] / V.V.Abramova, S. K. Abramov, V. V.Lukin, K. O. Egiazarian, J. T. Astola // EURASIP Journal on Image and Video Processing. - 2014. - No. 2014:3. - Available at: http://jivp.eurasipjournals.com/content/2014/1/3. 14.11.2018.

18. Airborne Visible/Infrared Imaging Spectrometer [Electronic Resource]. - Available at: http://aviris.jpl.nasa.gov. - 14.11.2018.
19. Imaging spectroscopy and the airborne visible/infrared imaging spectrometer (AVIRIS) [Text] / R. O. Green, M. L. Eastwood, C. M. Sarture, T. G. Chrien, M. Aronsson, B. J. Chippendale // Remote Sensing of Environment. - 1998. - No. 65. - P. 227 248.

20. The EO-1 Hyperion Imaging Spectrometer [Electronic Resource]. - Available at: https://eol. gsfc.nasa.gov/new/validationReport/Technology/TRW EO1\%20Papers_Presentations/10.pdf.-14.11.2018.

21. Hyperion, a space-based imaging spectrometer [Text] / J.S. Pearlman, P.S. Barry, C. C. Segal, J. Shepanski, D. Beiso, S. L. Carman // IEEE Transactions on Geoscience and Remote Sensing. - 2003. - No. 41(6). - P. 1160-1173.

22. Using inter-channel correlation in blind evaluation of noise characteristics in multichannel remote sensing images [Text] / V. V. Abramova, S. K. Abramov, V. V. Lukin, B. Vozel, K. Chehdi // ERS International Congress, Sept. 10-13, 2018. - Berlin, 2018. - Vol. 10004. - Article ID: 1000408. - 11 p.

23. Abramova, V. V. Iterative Method for Blind Evaluation of Mixed Noise Characteristics on Images [Text] / V. V. Abramova, S. K. Abramov, V. V. Lukin // Information and Telecommunication Sciences. - 2015. - Vol.6, No. 1. - P. 8-14.

24. Abramova, V. A Blind Method for Additive Noise Variance Evaluation Based on Homogeneous Region Detection Using the Fourth Central Moment Analysis [Text] / V. Abramova // Telecommunications and Radioengineering. - 2015. - No.74(18). P. $1651-1669$.

25. Image Informative Maps for Component-wise Estimating Parameters of Signal-Dependent Noise [Text] / M. Uss, B. Vozel, V. Lukin, K. Chehdi // Journal of Electronic Imaging. - 2013. - Vol. 22, No 1. - Article ID: 013019. $-7 p$.

\section{References}

1. Shovengerdt, R. A. Distantsionnoe zondirovanie. Modeli i metody obrabotki izobrazheniy [Remote Sensing. Models and Methods of Image Processing], Moskow, "Tekhnosfera" Publ., 2010. 560 p.

2. Popov, M., Stankevich, S., Markov, S. Integratsiya geterogennoy prostranstvennoy informatsii dlya resheniya zadach poiska nefti i gaza [Integration of heterogeneous spatial information in tasks of oil and gas search], Rossiyskiy nauchnyy elektronnyy zhurnal "Elektronnye biblioteki» - "Russian Digital Libraries", 2013, vol. 16, no. 2. Available at: http://www.elbib.ru (accessed 14.11.2018).

3. Kerekes, J. P. Optical Sensor Technology, The SAGE Handbook of Remote Sensing, London, UK, SAGE Publications, 2009, pp. 95 - 107.

4. Christophe, E. Hyperspectral Data Compression Tradeoff in Optical Remote Sensing, Advances in Signal Processing and Exploitation Techniques. 8th ed, Springer; Berlin Heidelberg, 2011, pp. 9-29. 
5. Bekhtin, Y. S. Adaptive wavelet codec for noisy image compression, Proceedings of the 9th EastWest Design and Test Symposium, Sevastopol, Ukraine, September 2011, pp. 184-188.

6. Image Classification Techniques in Remote Sensing. Available at: https://gisgeography.com/imageclassification-techniques-remote-sensing/ (accessed 1.10.2018).

7. Hu, Y., Chen, J., Pan, D., Hao, Z. Edge-guided image object detection in multiscale segmentation for high-resolution remotely sensed imagery, IEEE Transactions on Geoscience and Remote Sensing, 2016 , vol. 54, no. 8, pp. 4702-4711.

8. Zhong, P., Wang, R. Multiple-spectral-band CRFs for denoising junk bands of hyperspectral imagery, IEEE Transactions on Geoscience and Remote Sensing, 2013, no. 51(4), pp. 2269-2275.

9. Meola, J., Eismann, M. T., Moses, R. L., Ash, J. N. Modeling and estimation of signal-dependent noise in hyperspectral imagery, Applied Optics, 2011, no. 50(21), pp. 3829-3846.

10. Colom, M., Lebrun, M., Buades, A., Morel, J. M. A non-parametric approach for the estimation of intensity-frequency dependent noise, IEEE International Conference on Image Processing (ICIP), Paris, France, 27-30 October 2014, pp. 4261-4265.

11. Goosens, B., Pizurica, A., Wilfried, P. Removal of Correlated Noise by Modeling the Signal of Interest in the Wavelet Domain, IEEE Transactions on Image Processing, 2009, vol. 18, issue 6, pp. 1-14.

12. Cocianu, C, Stan, A. Neural Architectures for Correlated Noise Removal in Image Processing, Mathematical Problems in Engineering, 2016, vol. 2016, article ID: 6153749,14 p. Available at: https://www.hinda-wi.com/journals/mpe/2016/6153749/ (accessed 14.11.2018).

13. Liu, C., Szeliski, R., Kang, S. B., Zitnick, C. L., Freeman, W.T. Automatic estimation and removal of noise from a single image, IEEE Transactions on Pattern Analysis and Machine Intelligence, 2008, no. 30(2), pp. 299-314.

14. Gao, L., Du, Q., Zhang, B., Yang, W., Wu, Y. A comparative study on linear regression based noise estimation for hyperspectral imagery, IEEE Journal of Selected Topics in Applied Earth Observations and Remote Sensing, 2013, no. 6(2), pp. 488-498.

15. Jin, X., Xu, Z., Hirakawa, K. Noise parameter estimation for poisson corrupted images using variance stabilization transforms, IEEE Trans. Image Process., 2014, no. 23(3), pp. $1329-1339$.
16. Savant, R. V., Pradhan, D. Estimation of noise parameters for captured image [Electronic Resource], IEEE International Conference on Engineering and Technology (ICETECH), March 17 - 18, Coimbature, India. Available at: https://ieeexplore.ieee.org/document/7569405 (accessed 20.11.2018).

17. Abramova, V. V., Abramov, S. K., Lukin, V. V., Egiazarian, K. O., Astola, J. T. On required accuracy of mixed noise parameter estimation for image enhancement via denoising, EURASIP Journal on Image and Video Processing, 2014, no. 2014:3. Available at: http://jivp.eurasipjournals.com/con-tent/2014/1/3 (accessed 14.11.2018).

18. Airborne Visible/Infrared Imaging Spectrometer. Available at: http://aviris.jpl.nasa.gov (accessed 14.11.2018).

19. Green, R. O., Eastwood, M. L., Sarture, C. M., Chrien, T. G., Aronsson, M., Chippendale, B. J. Imaging spectroscopy and the airborne visible/infrared imaging spectrometer (AVIRIS), Remote Sensing of Environment, 1998, no. 65, pp. 227-248.

20.The EO-1 Hyperion Imaging Spectrometer. Available at: https://eo1.gsfc.nasa.gov/new/validationRport/Technology/TRW_EO1\%20Papers_Presentati ons/10.pdf. (accessed 14.11.2018).

21. Pearlman, J. S., Barry, P. S., Segal, C. C., Shepanski, J., Beiso, D., Carman, S. L. Hyperion, a space-based imaging spectrometer, IEEE Transactions on Geoscience and Remote Sensing, 2003, no. 41(6), pp. 1160-1173.

22. Abramova, V. V., Abramov, S. K., Lukin, V. V., Vozel, B., Chehdi, K. Using inter-channel correlation in blind evaluation of noise characteristics in multichannel remote sensing images, ERS International Congress, Berlin, Sept. 10-13, 2018, vol. 10004, article ID 1000408, 11 p.

23. Abramova, V. V., Abramov, S. K., Lukin, V. V. Iterative Method for Blind Evaluation of Mixed Noise Characteristics on Images, Information and Telecommunication Sciences, 2015, vol. 6, no. 1, pp. $8-14$.

24. Abramova, V. A Blind Method for Additive Noise Variance Evaluation Based on Homogeneous Region Detection Using the Fourth Central Moment Analysis, Telecommunications and Radioengineering, 2015, no. 74(18), pp. $1651-1669$.

25. Uss, M., Vozel, B., Lukin, V., Chehdi, K. Image Informative Maps for Component-wise Estimating Parameters of Signal-Dependent Noise, Journal of Electronic Imaging, 2013, vol. 22, no 1., Article ID: 013019, 7 p. 


\title{
СПЕЦИФІКА ОЦІНЮВАННЯ ХАРАКТЕРИСТИК СКЛАДНИХ ЗАВАД \\ З ВРАХУВАННЯМ МІЖКАНАЛЬНОЇ КОРЕЛЯЦІЇ НА ГІПЕРСПЕКТРАЛЬНИХ ЗОБРАЖЕННЯХ
}

\author{
А. С. Григор'єва, В. В. Абрамова, В. В. Лукін, \\ Н. В. Кожемякіна, К. Д. Абрамов
}

Предметом вивчення в статті $\epsilon$ скаттерограммний метод автоматичного оцінювання характеристик складних завад на багатоканальних зображеннях. Метою $є$ розгляд проблемних питань, пов'язаних з адаптацією даного методу для роботи з гіперспектральними зображеннями. Завдання: дослідити вплив способу формування груп канальних зображень, які підлягають сумісній обробці, на точність досліджуваного методу оцінювання та стабільність його роботи; сформулювати рекомендації щодо вибору зображень, які підлягають сумісній обробці, і способу об'єднання їх в групи. При вирішенні поставлених завдань були використані методи робастного оцінювання параметрів сигналів, а також методи спектрально-кореляційного і регресійного аналізу. Отримані наступні результати. Розглянуто три можливі способи формування груп з трьох канальних зображень: 1) спільна обробка зображень, які належать до сусідніх каналів; 2) спільна обробка зображень, що мають найвищі коефіцієнти взаємної кореляції; 3) спільна обробка зображень, що мають найнижчі коефіцієнти взаємної кореляції. Показано, що в разі, якщо коефіцієнти взаємної кореляції зображень, що входять в групу, низькі, а самі зображення характеризуються складною структурою, можливе суттєве зниження точності методу, аж до повної втрати його працездатності. При формуванні груп з сусідніх зображень і з зображень з найвищими коефіцієнтами міжканальної кореляції метод демонструє досить високі точність і стабільність, причому значення оцінок параметрів завад для цих варіантів відрізняються слабо. Висновки. Спосіб формування групи істотно впливає не тільки на точність, але й на працездатність даного методу оцінювання, і для забезпечення більш високої надійності його роботи доцільно об'єднувати в групу зображення з досить високими коефіцієнтами міжканальної кореляції. Однак оскільки точність методу в ситуаціях, коли в групу об'єднуються зображення, що належать сусіднім каналам, і зображення 3 найвищими коефіцієнтами кореляції, відрізняються незначно, з метою збереження високої швидкодії методу, групи зображень для їх спільної обробки рекомендується формувати із зображень, отриманих в сусідніх спектральних зонах.

Ключові слова: дистанційне зондування; гіперспектральні зображення; міжканальна кореляція; складні завади; автоматичне оцінювання характеристик завад

\section{ESTIMATION SPECIFICITY OF COMPLEX NOISE CHARACTERISTICS CONSIDER THE INTER-CHANNEL CORRELATION ON HYPERSPECTRAL IMAGES}

\section{A. S. Grigor'eva, V. V. Abramova, V. V. Lukin, K. D. Abramov, N. V. Kozhemyakina}

The article deals with the scatter-plot method of automatic evaluation of mixed noise characteristics in multichannel images. The aim is to solve the problematic issues associated with the adaptation of this method to hyperspectral images processing. The tasks to be solved are: to investigate the influence of the formation method of the jointly processed multichannel groups of images on the accuracy and stability of the aforementioned method; to formulate the recommendations on the choice of jointly processed images and the method of combination. The applied methods are the following: robust estimation of signal parameters, spectral, correlation and regression analysis. The following results were obtained. Three possible ways of groups forming of three channel images were considered: 1) joint processing of images belonging to adjacent channels; 2) joint processing of images with the highest cross-correlation coefficients; 3 ) joint processing of images with the lowest cross-correlation coefficients. It was defined that if the cross-correlation coefficients of images in the group are low, and the images have of complex structure, it is possible a significant reduction of the method accuracy, up to a complete loss of its working capacity. The method demonstrates sufficiently high accuracy and stability when the groups are formed of the neighbor channel images or of the images with the highest cross-correlation coefficients, and the values of the estimated noise parameters for these issues have no significant differences. Conclusions. The group formation method significantly affects not only the accuracy, but also the operability of the considered estimation method, and in order to increase the reliability of the method, it is appropriate to form groups of images with rather high levels of inter-channel correlation. However, since the accuracy of the method when groups are formed of the neighbor images and of images with the highest levels of cross-correlation have no significant differences, in order to maintain the high performance of the method, it is recommended to form groups of jointly processed images applying the images obtained in the neighbor spectral zones.

Keywords: remote sensing; hyperspectral images; inter-channel correlation; mixed noise; automatic evaluation of noise characteristics. 
Григорьева Алёна Сергеевна - магистрант кафедры информационно-коммуникационных технологий им. А. А. Зеленского, Национальный аэрокосмический университет им. Н. Е. Жуковского «Харьковский авиационный институт», Харьков, Украина.

Абрамова Виктория Валерьевна - канд. техн. наук, доцент кафедры информационнокоммуникационных технологий им. А. А. Зеленского, Национальный аэрокосмический университет им. Н. Е. Жуковского «Харьковский авиационный институт», Харьков, Украина.

Лукин Владимир Васильевич - д-р. техн. наук, профессор, заведующий кафедрой информационнокоммуникационных технологий им. А. А. Зеленского, Национальный аэрокосмический университет им. Н. Е. Жуковского «Харьковский авиационный институт», Харьков, Украина.

Абрамов Клавдий Данилович - канд. техн. наук, доцент кафедры информационнокоммуникационных технологий им. А. А. Зеленского, Национальный аэрокосмический университет им. Н. Е. Жуковского «Харьковский авиационный институт», Харьков, Украина.

Кожемякина Надежда Владимировна - ассистент кафедры информационно-коммуникационных технологий им. А. А. Зеленского, Национальный аэрокосмический университет им. Н. Е. Жуковского «Харьковский авиационный институт», Харьков, Украина.

Grigor'eva Alena Sergeevna - Master Degree student of Information-communication technologies named after A. A. Zelensky Chair, National Aerospace University "Kharkov Aviation Institute", Kharkov, Ukraine, e-mail: alenag077@gmail.com.

Abramova Victoriya Valerievna - PhD, Assistant Professor of Information-communication technologies named after A. A. Zelensky Chair, National Aerospace University "Kharkov Aviation Institute", Kharkov, Ukraine, e-mail: v.abramova@khai.edu.

ORCID Author ID: 0000-0001-5802-5858. Scopus Author ID: 55225531900. ResearchGate: Victoriya Abramova.

Lukin Vladimir Vasilievich - Doctor of Technical Science, Head of Information-communication technologies named after A. A. Zelensky Chair, National Aerospace University "Kharkov Aviation Institute", Kharkov, Ukraine, e-mail: v.lukin $@$,khai.edu.

ORCID Author ID: 0000-0002-1443-9685. Scopus Author ID: 7102438809. ResearchGate: Vladimir_Lukin2.

Abramov Klavdiy Danilovich - PhD, Assistant Professor of Information-communication technologies named after A. A. Zelensky Chair, National Aerospace University "Kharkov Aviation Institute", Kharkov, Ukraine, e-mail: k.abramov@khai.edu.

Kozhemyakina Nadezhda Vladimirovna - Assistant of Information-communication technologies named after A. A. Zelensky Chair, National Aerospace University "Kharkov Aviation Institute", Kharkov, Ukraine, e-mail: n.kozhemiakina@khai.edu. 